\title{
Analysis of post-operative efficacy and pharyngeal fistula healing in patients with laryngeal cancer treated with post-operative enteral nutrition support nursing combined with early oral feeding
}

\author{
AITAO LIN, JIN YE, ZHIYUAN WANG and PEI LI \\ Department of Otolaryngology-Head and Neck Surgery, The Third Affiliated Hospital, \\ Sun Yat-Sen University, Guangzhou, Guangdong 510630, P.R. China
}

Received October 23, 2018; Accepted December 31, 2019

DOI: $10.3892 / \mathrm{ol} .2020 .11503$

\begin{abstract}
The present study aimed to analyze the effects of post-operative enteral nutrition support nursing combined with early oral feeding on post-operative efficacy and pharyngeal fistula $(\mathrm{PF})$ healing in patients with laryngeal cancer (LC). A retrospective analysis of 133 patients with LC, who underwent laryngectomy between May 2014 and September 2016, was conducted. Of these patients, 61 patients (control group) were treated with enteral nutrition support nursing combined with nasogastric feeding, and 72 patients (observation group) were treated with enteral nutrition support nursing combined with early oral feeding. Levels of serum albumin (ALB), pre-albumin (PA), hemoglobin (Hb) and the lymphocyte count (LYM) were compared before and after surgery (7 and 14th post-operative days). The post-operative weight, upper arm muscle circumference (UAMC), triceps skinfold (TSF), post-operative infection, adverse reactions and PF healing were also measured. No differences were observed between the control and observation groups before surgery (all $\mathrm{P}>0.05)$. ALB , Hb, PA and LYM were significantly decreased in the control group on the 7 and 14th days post-operatively compared with those in the observation group (all $\mathrm{P}<0.05$ ). Five patients in the control group presented with PF during treatment, and seven patients in the observation group suffered from PF after surgery. No difference in the average healing time of PF, number of patients with post-operative infection
\end{abstract}

Correspondence to: Dr Jin Ye, Department of OtolaryngologyHead and Neck Surgery, The Third Affiliated Hospital, Sun Yat-Sen University, 600 Tianhe Road, Tianhe, Guangzhou, Guangdong 510630, P.R. China

E-mail: yejin876m@163.com

Abbreviations: PF, pharyngeal fistula; LC, laryngeal cancer; ALB, albumin; PA, pre-albumin; Hb, hemoglobin; LYM, lymphocyte count; UAMC, upper arm muscle circumference; TSF, triceps skinfold; TNM, tumor-node-metastasis

Key words: enteral nutrition support, oral feeding, LC, PF and adverse reactions were observed between the two groups (all $\mathrm{P}>0.05$ ). The weight and UAMC in the observation group, 14 days post-operatively, were significantly higher than those before surgery in the same patients and in the control group (all $\mathrm{P}<0.05)$, whereas no difference in TSF $(\mathrm{P}>0.05)$ was observed. No differences in weight, UAMC and TSF were revealed in the control group before surgery and on the 14th day post-operatively (all $\mathrm{P}>0.05$ ). Thus, post-operative enteral nutrition support nursing combined with early oral feeding resulted in significant improvement in the post-operative efficacy of patients with LC and had no effect on PF healing.

\section{Introduction}

As a common malignant tumor in the head and neck, laryngeal cancer (LC) is more common in males over the age of 40 and has a higher incidence rate in Asia compared with other regions (1). The incidence of LC accounts for $1 \%$ of malignant tumors in the whole body and $>5 \%$ of head and neck cancer cases. Despite its low incidence, LC is a threat to patient quality of life if not treated in a timely manner (2). Current treatment methods include surgery, radiotherapy, chemotherapy and immunotherapy $(3,4)$. In clinical practice, patients with LC are often treated with a combination of different methods; however, surgical excision remains the main clinical method for treating LC (5). Advancements in laryngectomy procedures have improved the initial survival rate of patients with LC, and preserve laryngeal function, which has an impact on quality of life (6).

Pharyngeal fistula (PF), one of the complications that occurs after laryngectomy, refers to the formation of an abscess cavity after surgical resection. PF originates from the saliva or pus, stored under the subcutaneous or incision tissue that connects the hypopharynx, esophagus and skin to the sinus tract, through which saliva or food flows out (7). PF does not only prolong the treatment time of patients, but also impacts the financial burden of their family (8). Due to the influence of laryngectomy on deglutition, patients cannot eat within a short period of time post-surgery, which leads to slow recovery, malnutrition and post-operative infection (9). Enteral nutrition support is provided to patients using a nasogastric feeding tube (NFT), 
the main method of nutrition support after laryngectomy, which has a beneficial effect on post-operative recovery; however, patients using NFT long-term are prone to malnutrition, which hinders the growth of granulation tissue (10).

A previous study demonstrated that early oral feeding provides nutritional support for patients with LC. Furthermore, the coordination between the muscle groups of the oral floor and neck during oral feeding is a form of exercise, which promotes the blood circulation and wound healing. Moreover, the secretion of saliva and washing of the food passage during feeding cleans the inner wall of the fistula and promotes the maintenance of normal bacterial flora in the oral and pharyngeal cavity. This allows the normal bacterial flora to inhibit the growth of pathogenic bacteria in the fistula and decreases inflammation (11). However, the time to commence oral feeding and whether it has an impact on PF treatment is unknown.

In the present study, the effect of post-operative enteral nutrition support nursing combined with early oral feeding (following a laryngectomy) on the post-operative efficacy and PF healing of patients with LC was analyzed.

\section{Materials and methods}

Patients. The clinical data of 133 patients with LC who underwent a laryngectomy in The Third Affiliated Hospital, Sun Yat-Sen University, between May 2014 and September 2016, were retrospectively analyzed. All patients were diagnosed with LC, based on the pathology of biopsies, and tumor-node-metastasis (TNM) staging was performed on patients in accordance with The American Joint Committee on Cancer criteria (12). Patients $(n=133)$ were divided into two groups: Control and observation groups. The control group included 61 patients (39 males and 22 females; mean age, 55.84 \pm 8.64 years; age range, $45-68$ years). The observation group included 72 patients (49 males and 23 females; mean age, $56.25 \pm 9.35$ years; age range, $42-69$ years). The present study was approved by the Medical Ethics Committee of the Third Affiliated Hospital, Sun Yat-Sen University, and written informed consent was obtained from the participants.

Inclusion and exclusion criteria. The inclusion criteria were as follows: (i) Patients $>18$ years; (ii) patients who had not received preoperative radiotherapy and chemotherapy; and (iii) patients who complied with the treatment administered. The exclusion criteria were as follows: Patients with (i) a family history of genetic diseases; (ii) close relatives with history of LC; (iii) autism, memory disorders, hearing disorders and physical disabilities; and (iv) recurrence and metastasis post-surgery.

Feeding. Patients in the control and observation groups were observed for a total of 14 days. Patients in the control group were fed through a NFT for 14 days. In the observation group, the NFT was removed on the 7th day of nasogastric feeding, and oral feeding was initiated if no PF appeared within 7 days. Patients in the observation group were also trained to perform dry swallowing (swallowing without saliva) six times a day for 10-15 min each time, depending on their condition. In the early stage of feeding, patients in the observation group were mainly fed soft food, and gradually transitioned to normal food (from small and soft food to large and hard food), under the guidance and training of nursing staff. Mouthwash (0.9\% sodium chloride solution; Hunan Jinjian Pharmaceutical Co., Ltd.) was used to wash after feeding. Patients in both groups received routine cefuroxime infusions $(1.5 \mathrm{~g} / 8 \mathrm{~h}$; Sinopharm Zhijun Pharmaceutical Co., Ltd.,), for prophylactic anti-infective treatment, as well as compound electrolytes and nutrient solution (intravenous drip (ivgtt), each $1,000 \mathrm{ml}$ contains $5.26 \mathrm{~g}$ sodium chloride, $5.02 \mathrm{~g}$ sodium gluconate, $3.68 \mathrm{~g}$ sodium acetate, $0.37 \mathrm{~g}$ potassium chloride and $0.30 \mathrm{~g}$ magnesium chloride; China Sichuan Kelun Pharmaceutical Co., Ltd.).

Nursing. Patients in the control and observation groups received nursing care as follows. Routine nursing consisted of nurses: i) Visiting the patients every hour to observe their condition; ii) monitoring the vital signs of the patients, depending on their condition; and iii) cleaning the wound as appropriate and maintaining its sterility. Nutrition support nursing consisted of the following: i) Strictly controlling the rate of nutrient infusion to $25 \mathrm{ml} / \mathrm{h}$ in the first $6 \mathrm{~h}$ on the 1st post-operative day to avoid the occurrence of gastric retention; ii) observing the clinical manifestations in patients and increasing the infusion rate to $30-60 \mathrm{ml} / \mathrm{h}$ if the patient appeared comfortable; iii) adjusting the patients to a semi-reclining position to avoid regurgitation of nutrients and maintaining the gastric tube fixed and unobstructed; iv) maintaining the temperature of the nutrient solution between $38-40^{\circ} \mathrm{C}$ using a heater; and v) rinsing the nasogastric tube with warm water after infusion and once every $4 \mathrm{~h}$ during infusion.

PF processing. In case of PF formation, the necrotic tissues were cleaned and drained immediately. The airway-associated care was strengthened, by maintaining the ventilation of the patient and using auxiliary equipment, when breathing was difficult. The wound was cleaned using saline $(0.9 \%$ sodium chloride solution; Hunan Jinjian Pharmaceutical Co., Ltd.) and hydrogen peroxide (3\%). An in-house antibiotic gauze (applying $400 \mathrm{mg}$ of different antibiotic ointment evenly on the sterile gauze block with sterile scraper to cover the PF position) was used to fill the wound with pressure dressing, depending on the susceptibility of the patient to infection indicated by their test results of drug sensitivity. Patients who did not respond to medication were additionally treated with surgery.

Detectionmethod.Serum albumin (ALB) and pre-albumin (PA) were detected with a Beckman 5800 system (Beckman Coulter, Inc.). ALB was detected by bromocresol green method. The principle of this method is that bromocresol green reacts with albumin to form a green complex and then the absorbance is read at $600 \mathrm{~nm}$ (13). The immunoturbidimetric method was used to detect PA. The principle of this detection method is that the antibody (prealbumin PA; cat. no. C079-h; incubated at $37^{\circ} \mathrm{C}$ for $60 \mathrm{~min}$; China Jilin Changchun Huili Biotechnology Co., Ltd.) reacts with the soluble antigen to form an immune complex, which becomes the particle suspended in the reaction solution. The absorbance is read at $340 \mathrm{~nm}$ for the main wave and at $750 \mathrm{~nm}$ for the secondary wave. The kit was purchased from Beijing Jiuqiang Biotechnology Co., Ltd. (cat. no. GS631M) and was used according to the manufacturer's instructions. Hemoglobin ( $\mathrm{Hb})$ and lymphocyte count (LYM) were measured with automatic blood routine analyzer XT-2000i (5 classification; Sysmex Corporation). 
Table I. Characteristics of patients in the control $(n=61)$ and observation $(n=72)$ groups.

\begin{tabular}{|c|c|c|c|c|}
\hline Characteristics & Control group, n (\%) & Observation group, n (\%) & $t / \chi^{2}$ & P-value \\
\hline Sex & & & 0.251 & 0.617 \\
\hline Male & $39(63.93)$ & $49(68.06)$ & & \\
\hline Female & $22(36.04)$ & $23(31.94)$ & & \\
\hline Age, years & & & 0.006 & 0.937 \\
\hline$\geq 55$ & $36(59.02)$ & $42(58.33)$ & & \\
\hline$<55$ & $25(40.98)$ & $30(41.67)$ & & \\
\hline $\mathrm{BMI}, \mathrm{kg} / \mathrm{m}^{2}$ & $22.36 \pm 1.25$ & $22.84 \pm 1.84$ & 1.727 & 0.087 \\
\hline Smoking habit & & & 0.453 & 0.501 \\
\hline Yes & $39(63.93)$ & $50(69.44)$ & & \\
\hline No & $22(36.04)$ & $22(30.56)$ & & \\
\hline Hypertension & & & 1.320 & 0.251 \\
\hline Yes & $50(81.97)$ & $53(73.61)$ & & \\
\hline No & $11(18.03)$ & $19(26.39)$ & & \\
\hline Diabetes mellitus & & & 0.226 & 0.634 \\
\hline Yes & $40(65.57)$ & $50(69.44)$ & & \\
\hline No & $21(34.43)$ & $22(30.56)$ & & \\
\hline TNM stage & & & 1.114 & 0.291 \\
\hline $\mathrm{T} 1$ & $30(49.18)$ & $42(58.33)$ & & \\
\hline $\mathrm{T} 2$ & $31(50.82)$ & $30(41.67)$ & & \\
\hline Surgical program & & & 3.957 & 0.047 \\
\hline Total laryngectomy & $7(11.48)$ & $18(25.00)$ & & \\
\hline Partial resection & $54(88.52)$ & $54(75.00)$ & & \\
\hline
\end{tabular}

BMI, body mass index; TNM, tumor node metastasis.

Outcome measures. The primary outcome measures were ALB, PA, Hb, LYM levels and PF healing time. The secondary outcome measures were occurrences of post-operative infection, adverse reactions, weight, upper arm muscle circumference (UAMC) and triceps skinfold (TSF). The primary and secondary outcomes were measured before surgery and on the 7 and the 14th post-operative days.

Statistical analysis. All statistical analyses were performed using SPSS software (version 20.0; IBM Corp.) and graphs were drawn using GraphPad Prism software (version 7.0; GraphPad Software, Inc.). Measurement data are expressed as the mean \pm SD. Data expressed as rate (\%) were assessed by the $\chi^{2}$ and Fisher exact tests. Independent or paired t-test was used for comparison between and within two groups, respectively. Repeated measures ANOVA was used for analysis between more than two groups; Bonferroni correction was used for post hoc test. $\mathrm{P}<0.05$ was considered to indicate a statistically significant difference.

\section{Results}

Patient characteristics. Comparisons of the clinical data, between patients in the control and observation groups, revealed no significant differences in the sex, age, BMI, smoking history, hypertension history, diabetes history and TNM stage (all P>0.05; Table I). The surgical treatment plans of two groups were significantly different $(\mathrm{P}<0.05)$.
Changes in primary outcomes of patients with LC. Comparisons between the two groups revealed no significant differences in ALB, PA, Hb and LYM before surgery (all P>0.05). ALB, $\mathrm{Hb}$ and PA levels were significantly decreased, whereas LYM was significantly increased, on the 7th post-operative day in both groups compared with those before surgery (all $\mathrm{P}<0.05$ ). ALB, Hb, PA levels and the LYM in the control group were significantly decreased on the 7th post-operative day compared with those of the observation group (all $\mathrm{P}<0.05$ ). On the 14th post-operative day, PA levels were significantly decreased, whereas LYM was significantly increased, in both groups compared with those before surgery (all $\mathrm{P}<0.05$ ). There were no differences in ALB and $\mathrm{Hb}$ levels in the observation group on the 14th post-operative day compared with those before surgery (both $\mathrm{P}>0.05$ ). Meanwhile, ALB and $\mathrm{Hb}$ levels in the control group were significantly decreased on the 14th post-operative day compared with those before surgery (both $\mathrm{P}<0.05$ ). Finally, ALB, Hb, PA levels and LYM were significantly decreased in the control group on the 14th post-operative day compared with those in the observation group (all $\mathrm{P}<0.05$; Figs. 1-4).

PF healing. In the present study, patients presented with PF in both groups, including five patients in the control group and seven patients in the observation group. There were no differences in the average healing times between the control (7.54 \pm 2.35 days) and the observation $(7.25 \pm 2.01$ days) groups, following the treatment for PF ( $\mathrm{P}>0.05$; data not shown). 


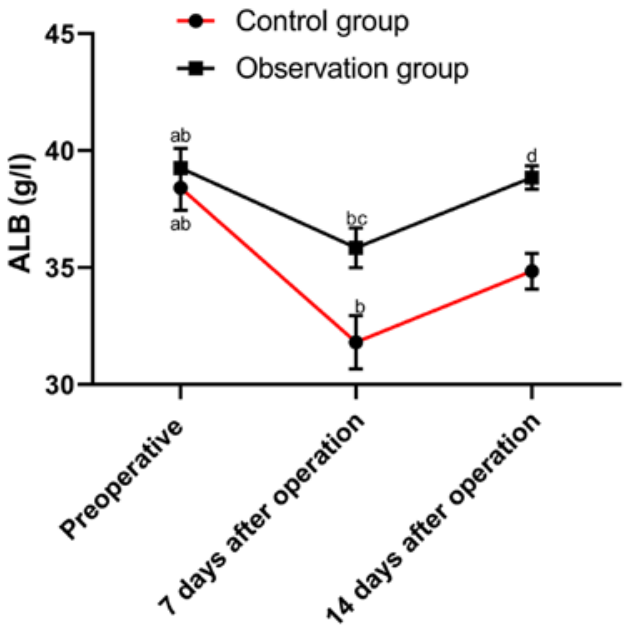

Figure 1. Change in ALB levels before and after surgery in the two groups. Repeated measures ANOVA followed by Bonferroni's correction was used for analysis. 'a' indicates a significant difference compared with 7 days after treatment within the group $(\mathrm{P}<0.05)$. ' $\mathrm{b}$ ' indicated a significant difference compared with 14 days after treatment within the group $(\mathrm{P}<0.05)$. ' $\mathrm{c}$ ' indicates significant difference between group comparison at 7 days after treatment $(\mathrm{P}<0.05)$. ' $\mathrm{d}$ ' indicates a significant difference between group comparison at 14 days $(\mathrm{P}<0.05)$. ALB, albumin.

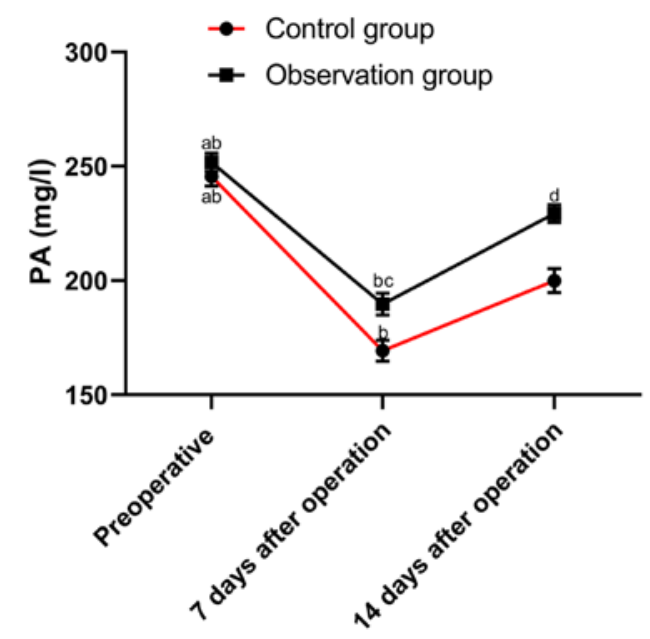

Figure 2. Change in PA levels before and after surgery in the two groups. Repeated measures ANOVA followed by Bonferroni's correction was used for analysis. 'a' indicates a significant difference compared with 7 days after treatment within the group $(\mathrm{P}<0.05)$. ' $b$ ' indicates a significant difference compared with 14 days after treatment within the group $(\mathrm{P}<0.05)$. ' $\mathrm{c}$ ' indicates a significant difference between group comparison at 7 days after treatment $(\mathrm{P}<0.05)$. 'd' indicates a significant difference between group comparisons at 14 days $(\mathrm{P}<0.05)$. $\mathrm{PA}$, prealbumin.

Analysis of adverse reactions. The number of patients with post-operative infection, $\mathrm{PF}$, diarrhea, bloating, nausea and vomiting in the control and observation groups during treatment was not statistically different (all $\mathrm{P}>0.05$; Table II).

Nutritional status indicators. Before surgery, there were no differences in the weight, UAMC and TSF of patients between the control and observation groups (all $\mathrm{P}>0.05$ ). On the 14th post-operative day, the weight and UAMC in the observation group were significantly higher than those before surgery (both $\mathrm{P}<0.05)$, whereas no difference in TSF was observed $(\mathrm{P}>0.05)$.

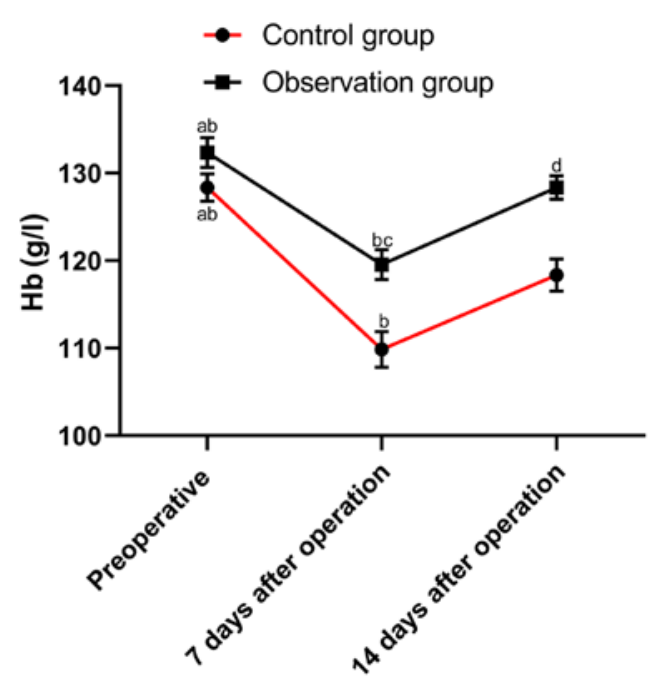

Figure 3. Change in $\mathrm{Hb}$ levels before and after surgery in the two groups. Repeated measures ANOVA followed by Bonferroni's correction was used for analysis. ' $a$ ' indicated that there was a significant difference compared with 7 days after treatment within the group $(\mathrm{P}<0.05)$. ' $\mathrm{b}$ ' indicates a significant difference compared with 14 days after treatment within the group $(\mathrm{P}<0.05)$. ' $\mathrm{c}$ ' indicates a significant difference between group comparison at 7 days after treatment $(\mathrm{P}<0.05)$. 'd' indicates a significant difference between group comparison at 14 days $(\mathrm{P}<0.05)$. Hb, hemoglobin.

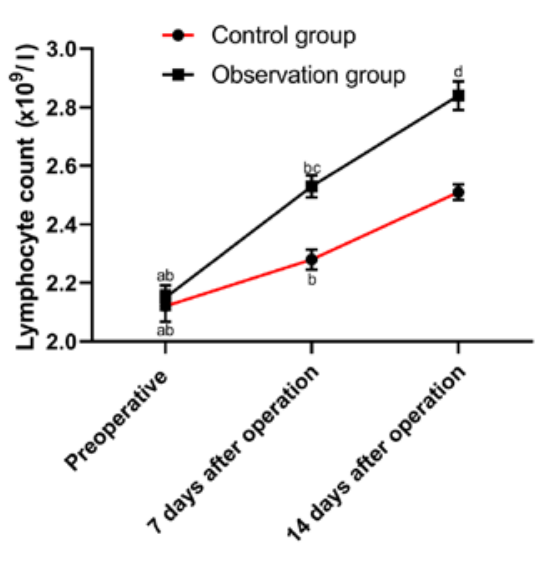

Figure 4. Change in the lymphocyte count before and after surgery in two groups. Repeated measures ANOVA followed by Bonferroni's correction was used for analysis. 'a' indicates a significant difference compared with 7 days after treatment within the group $(\mathrm{P}<0.05)$. ' $\mathrm{b}$ ' indicates a significant difference compared with 14 days after treatment within the group $(\mathrm{P}<0.05)$. 'c' indicates a significant difference between group comparison at 7 days after treatment $(\mathrm{P}<0.05)$. 'd' indicates a significant difference between group comparison at 14 days $(\mathrm{P}<0.05)$.

In the control group, no differences in weight, UAMC and TSF were demonstrated on the 14th post-operative day compared with those before surgery $(\mathrm{P}>0.05)$. On the 14 th post-operative day, the weight and UAMC in the control group were significantly lower than those of the observation group (all $\mathrm{P}<0.05$ ), while there was no difference in TSF ( $\mathrm{P}>0.05$; Table III).

\section{Discussion}

With the development of social economy and industry, the number of smokers is increasing, which leads to a number of 
Table II. Analysis of adverse reactions in control and observation groups, n (\%).

\begin{tabular}{lcccccc}
\hline Group & Infection & PF & Diarrhea & Bloating & Nausea & Vomiting \\
\hline Control group & $2(3.28)$ & $5(8.20)$ & $2(3.28)$ & $1(1.64)$ & $3(4.92)$ & $2(3.28)$ \\
Observation group & $1(1.39)$ & $7(9.72)$ & $2(2.78)$ & 0 & $1(1.39)$ & $2(2.78)$ \\
P-value & 0.999 & 0.999 & 0.999 & 0.459 & 0.333 & 0.999 \\
\hline
\end{tabular}

PF, pharyngeal fistula.

Table III. Nutritional status indicators in control and observation groups before and after surgery.

\begin{tabular}{lccccc}
\hline & \multicolumn{2}{c}{ Control group } & & \multicolumn{2}{c}{ Observation group } \\
\cline { 2 - 3 } \cline { 5 - 5 } Indicators & Before surgery & Preoperative 7th day & & Before surgery & Preoperative 14th day \\
\hline Weight, $\mathrm{kg}$ & $50.65 \pm 8.64$ & $51.35 \pm 9.53$ & & $51.38 \pm 10.84$ & $55.64 \pm 8.62^{\mathrm{a}}$ \\
UAMC, $1 / \mathrm{cm}$ & $20.53 \pm 4.86$ & $19.84 \pm 4.63$ & & $20.84 \pm 4.66$ & $22.81 \pm 5.37^{\mathrm{a}}$ \\
TSF, $\mathrm{d} / \mathrm{mm}$ & $7.62 \pm 3.20$ & $7.25 \pm 2.15$ & & $7.54 \pm 2.93$ & $7.15 \pm 2.11$ \\
\hline
\end{tabular}

${ }^{\text {a }} \mathrm{Pe}<0.05$ vs. the same group before surgery. UAMC, upper arm muscle circumference; TSF, triceps skinfold.

types of respiratory diseases (14). LC is a common malignant tumor in the head and neck, most common in males, and is associated with poor living conditions and smoking (15). As an important organ in the respiratory process, the larynx has three functions: Respiration, deglutition and vocalization. The larynx also plays a role in protecting the lower respiratory tract (16).

At present, the treatment of early LC is primarily surgical; however, the direct exposure of the wound to the air after surgery and the lack of laryngeal protection easily leads to infection and complications (17). Furthermore, a previous study has shown that malnutrition is an important cause of poor wound healing following surgery for head and neck cancer (18).

Post-operative enteral nutrition support is an important type of post-operative nutritional care for patients with LC, and ensures the delivery of nutrients via a feeding tube to support their daily metabolism and is beneficial for their prognosis (19). A recent study demonstrated that early oral feeding provides nutrition for patients, and has no effect on PF healing; furthermore, this type of nutritional intake promotes early post-operative rehabilitation, following resection of tumors in patients with colorectal cancer (20). Therefore, the present study investigated the effects of post-operative enteral nutrition support nursing combined with early oral feeding on the post-operative efficacy and PF healing time in patients with LC and identified the optimal treatment and nursing options for the clinical setting.

In the present study, ALB, PA, Hb levels and LYM were measured before and after surgery in the control and observation groups. ALB maintains blood colloid osmotic pressure, inhibits platelet aggregation and eliminates free radicals in the body, and is a good indicator of general health (21). The concentration of serum PA reflects the nutritional status of the body, which is often used as an important indicator of changes in post-operative nutritional status. Blood $\mathrm{Hb}$ levels partially reflect the nutritional status of patients $(22,23)$. Furthermore, when the immunity of a patient is weakened, LYM is decreased and the patient becomes prone to infections, inhibiting wound healing (24).

The present study demonstrated that the levels of ALB, Hb, PA and LYM in the control group were significantly reduced on the 7 and 14th post-operative days compared with those in the observation group. This indicated improvement in the post-operative condition of the patients, following nutrition support nursing combined with early oral feeding, in contrast to the combination with nasogastric feeding. This finding was consistent with the study by Süslü and Şefik Hoşal (25), which reported ameliorative effects on the immune system and nutritional status of patients following early oral feeding. Furthermore, the present study demonstrated no difference in the average PF healing time between the two groups. However, it was speculated that the cause of PF in patients may be associated with the surgical method and the age of the patient. A study by Seven et al (26) indicated there was no effect on PF in patients following oral feeding on the 7th post-operative day. Additionally, a study by Kishikova and Fleming (27) also demonstrated that early oral feeding had no effect on PF healing. The aforementioned studies are consistent with the results obtained in the present study. Statistical analysis on the adverse reactions of patients in the two groups demonstrated no differences between the two groups post-operatively, which might be influenced by the small sample size.

Finally, the weight, UAMC and TSF of patients in the two groups were compared. UAMC and TSF were important indicators that determine the development of subcutaneous fat and total protein storage (28). It was demonstrated that there were no changes in the weight, UAMC and TSF in the control group following surgery. In the observation group, no differences were observed in TSF before and after surgery. Meanwhile, the 
weight and UAMC in the observation group were significantly increased after surgery compared with those before surgery and those in the control group. These results suggested that early oral feeding exhibited beneficial effects compared with enteral nutrition support nursing combined with nasogastric feeding.

However, the short observation time, lack of long-term follow-up, lack of complete understanding of the details of the patient's change in condition, and the small sample size are limitations of the present study. Moreover, the scope of surgical resection and the chemoradiation regimens of phase 1 and 2 were not consistent, which may affect the results. In addition, the main cause of PF in patients is unclear. Therefore, future studies including a longer follow-up time and larger sample size are warranted. Furthermore, patient grouping according to the scope of surgical resection is required and risk factors of PF require further investigation. This will allow the investigation of independent risk factors and the verification of the findings from the present study.

In conclusion, post-operative enteral nutrition support nursing combined with early oral feeding significant improved the post-operative outcomes of patients with LC and had no effect on PF healing.

\section{Acknowledgements}

Not applicable.

\section{Funding}

No funding was received.

\section{Availability of data and materials}

The datasets used and/or analyzed during the current study are available from the corresponding author on reasonable request.

\section{Authors' contributions}

AL was the guarantor of integrity of the entire study, and contributed to the study concepts, study design, data acquisition and manuscript preparation. JY was responsible for data collection and analysis as well as manuscript writing and revision. ZW contributed to the experimental studies, literature research and clinical studies. PL performed the statistical analysis and data analysis. The final version of the manuscript has been read and approved by all authors.

\section{Ethical approval and consent to participate}

The present study was approved by the Ethics Committee of the Third Affiliated Hospital, Sun Yat-Sen University. All patients provided informed consent.

\section{Patient consent for publication}

Not applicable.

\section{Competing interests}

The authors declare that they have no competing interests.

\section{References}

1. Xuan L, Qu L, Zhou H, Wang P, Yu H, Wu T, Wang X, Li Q, Tian L, Liu M and Sun Y: Circular RNA: A novel biomarker for progressive laryngeal cancer. Am J Transl Res 8: 932-939, 2016.

2. Siegel R, Ma J, Zou Z and Jemal A: Cancer statistics, 2014. CA Cancer J Clin 64: 9-29, 2014.

3. Yang R: Corruption in China's higher education system: A malignant tumor. US-China Educ Rev 2: 18-21, 2015.

4. Metelmann HR, Woedtke TV, Kai M, Hyckel P and Podmelle F: Immunotherapy and immunosurveillance of oral cancers: Perspectives of plasma medicine and mistletoe. Cancer Immunol: 313-318, 2015.

5. Timme DW, Jonnalagadda S, Patel R, Rao K and Robbins KT: Treatment selection for T3/T4a laryngeal cancer: Chemoradiation versus primary surgery. Ann Otol Rhinol Laryngol 124: 845-851, 2015.

6. Elegbede AI, Rybicki LA, Adelstein DJ, Kaltenbach JA Lorenz RR, Scharpf J and Burkey BB: Oncologic and functional outcomes of surgical and nonsurgical treatment of advanced squamous cell carcinoma of the supraglottic larynx. JAMA Otolaryngol Head Neck Surg 141: 1111-1117, 2015.

7. Dedivitis RA, Aires FT, Cernea CR and Brandão LG: Pharyngocutaneous fistula after total laryngectomy: Systematic review of risk factors. Head Neck 37: 1691-1697, 2015.

8. Goepfert RP, Hutcheson KA, Lewin JS, Desai NG, Zafereo ME, Hessel AC, Lewis CM, Weber RS and Gross ND: Complications, hospital length of stay, and readmission after total laryngectomy. Cancer 123: 1760-1767, 2016.

9. Stankovic M, Milisavljevic D, Zivic M, Stojanov D and Stankovic P: Primary and salvage total laryngectomy. Influential factors, complications, and survival. J BUON 20: 527-539, 2015.

10. van der Linden NC, Kok A, Leermakers-Vermeer MJ, de Roos NM, de Bree R, van Cruijsen $\mathrm{H}$ and Terhaard $\mathrm{CH}$ : Indicators for enteral nutrition use and prophylactic percutaneous endoscopic gastrostomy placement in patients with head and neck cancer undergoing chemoradiotherapy. Nutr Clin Pract 32: 225-232, 2017.

11. Hay A, Pitkin L and Gurusamy K: Early versus delayed oral feeding in patients following total laryngectomy. Adv Otolaryngol 2014: P1-P10, 2015.

12. Yang X, Ren GX, Zhang CP, Zhou GY, Hu YJ, Yang WJ, Guo W, Li J and Zhong LP: Neck dissection and post-operative chemotherapy with dimethyl triazeno imidazole carboxamide and cisplatin protocol are useful for oral mucosal melanoma. Bmc Cancer 10: 623, 2010.

13. Lang L, Li X, Luo X, Du J, Huang Y, Cao D and Li M: Comparison of test results and performances of hitachi 7600 and beckman olympus 5800 automatic biochemical analyzers. Int J Clin Exp Med 11: 5095-5102, 2018.

14. Holland AE, Spruit MA, Troosters T, Puhan MA, Pepin V, Saey D, McCormack MC, Carlin BW, Sciurba FC, Pitta F, et al: An official European Respiratory Society/American Thoracic Society technical standard: Field walking tests in chronic respiratory disease. Eur Respir J 44: 1428-1446, 2014.

15. Møller PK, Tolstrup JS, Olsen MH, Dalton SO, Overgaard J and Johansen J: Predictors of continuous tobacco smoking in a clinical cohort study of Danish laryngeal cancer patients smoking before treated with radiotherapy. Acta Oncologica 54: 685-692, 2015.

16. Mattsson P, Hydman J and Svensson M: Recovery of laryngeal function after intraoperative injury to the recurrent laryngeal nerve. Gland Surg 4: 27-35, 2015.

17. Sotirović J, Šuljagić V, Baletić N, Pavićević L, Bijelić D, Erdoglija M, Perić A and Soldatović I: Risk factors for surgical site infection in laryngeal cancer surgery. Acta Clin Croat 54: 57-64, 2015.

18. Ackerman D, Laszlo M, Provisor A and Yu A: Nutrition management for the head and neck cancer patient. Cancer Treat Res 174: 187-208, 2018.

19. Wiggs CM: Case study: Baby John-nursing reflections on moral angst. Nurs Ethics 18: 606-612, 2011.

20. Sousa AA, Porcaro-Salles JM, Soares JM, Meyer de Moraes G, Souza Silva G, Abreu Sepulcri R, Rezende Carvalho J and Savassi-Rocha PR: Tolerance of early oral feeding in patients subjected to total laryngectomy. Head Neck 38 (Suppl 1): E643-E648, 2016.

21. Huang J, Lin L, Sun D, Chen H, Yang D and Li Q: ChemInform abstract: Bio-inspired synthesis of metal nanomaterials and applications. Chem Soc Rev 44: 6330-6374, 2015. 
22. Isshiki M, Hirayama S, Ueno T, Ito M, Furuta A, Yano K, Yamatani K, Sugihara M, Idei M and Miida T: Apolipoproteins C-II and C-III as nutritional markers unaffected by inflammation. Clin Chim Acta 481: 225-230, 2018.

23. Minekus M, Alminger M, Alvito P, Balance $S$, Bohn T, Bourlieu C, Carrière F, Boutrou R, Corredig M, Dupont D, et al: A standardised static in vitro digestion method suitable for food-an international consensus. Food Funct 5: 1113-1124, 2014.

24. Iwata E, Shigematsu H, Yamamoto Y, Tanaka M, Okuda A, Morimoto Y, Masuda K, Nakajima H, Koizumi M and Tanaka Y: Lymphocyte count at 4 days postoperatively: A reliable screening marker for surgical site infection following posterior lumbar decompression surgery. Spine (Phila Pa 1976) 43: E1096-E1101, 2018.

25. Süslü N and Sefik Hosal A: Early oral feeding after total laryngectomy: Outcome of 602 patients in one cancer center. Auris Nasus Larynx 43: 546-550, 2016.
26. Seven $\mathrm{H}$, Calis AB and Turgut S: A randomized controlled trial of early oral feeding in laryngectomized patients. Laryngoscope 113: 1076-1079, 2003.

27. Kishikova L and Fleming JC: Oral feeding following laryngectomy: Early or delayed? Int J Surg 12: 1137-1140, 2014.

28. Anoop S, Misra A, Bhatt SP, Gulati S, Mahajan H and Prabakaran G: High plasma glucagon levels correlate with waist-to-hip ratio, suprailiac skinfold thickness, and deep subcutaneous abdominal and intraperitoneal adipose tissue depots in nonobese Asian Indian males with type 2 diabetes in North India. J Diabetes Res 2017: 2376016, 2017.

(i) (9) This work is licensed under a Creative Commons Attribution-NonCommercial-NoDerivatives 4.0 International (CC BY-NC-ND 4.0) License. 